\title{
The XMM-Newton survey of the Small Magellanic Cloud: a new X-ray view of the symbiotic binary SMC 3
}

\author{
R. Sturm ${ }^{1}$, F. Haberl ${ }^{1}$, J. Greiner ${ }^{1}$, W. Pietsch ${ }^{1}$, N. La Palombara ${ }^{2}$, M. Ehle ${ }^{3}$, M. Gilfanov ${ }^{4,5}$, \\ A. Udalski ${ }^{6}$, S. Mereghetti ${ }^{2}$, and M. Filipović ${ }^{7}$
}

1 Max-Planck-Institut für extraterrestrische Physik, Giessenbachstraße, 85748 Garching, Germany
e-mail: rsturm@mpe.mpg. de
2 INAF, Istituto di Astrofisica Spaziale e Fisica Cosmica Milano, via E. Bassini 15, 20133 Milano, Italy
3 XMM-Newton Science Operations Centre, ESAC, ESA, PO Box 50727, 28080 Madrid, Spain
4 Max-Planck-Institut für Astrophysik, Karl-Schwarzschild-Str.1, 85741 Garching, Germany
5 Space Research Institute, Russian Academy of Sciences, Profsoyuznaya 84/32, 117997 Moscow, Russia
6 Warsaw University Observatory, Aleje Ujazdowskie 4, 00-478 Warsaw, Poland
7 University of Western Sydney, Locked Bag 1797, Penrith South DC, NSW1797, Australia

Received 21 October 2010 / Accepted 31 March 2011

ABSTRACT

\begin{abstract}
Context. The XMM-Newton survey of the Small Magellanic Cloud (SMC) was performed to study the population of X-ray sources in this neighbouring galaxy. During one of the observations, the symbiotic binary SMC 3 was found at its highest X-ray luminosity as observed until now.

Aims. In SMC 3 wind accretion from a giant donor star onto a white dwarf is believed to cause steady hydrogen burning on the white dwarf surface, making such systems candidates for supernova type Ia progenitors. It was suggested that the X-ray source is eclipsed every $\sim 4.5$ years by both the companion star and its stellar wind to explain the large X-ray variability seen in ROSAT data. We use the available X-ray data to test this scenario.

Methods. We present the $\sim 20$ year X-ray light curve of SMC 3 and study the spectral evolution as seen with XMM-Newton/EPIC-pn to investigate possible scenarios which can reproduce the high X-ray variability.

Results. We did not find any significant variations in the photo-electric absorption, as would be expected during eclipse ingress and egress. Instead, the X-ray spectra from different intensity levels, when modelled by black-body emission, can be better explained by variations either in normalisation (by a factor of $\sim 50$ ) or in temperature ( $k T$ between $24 \mathrm{eV}$ and $34 \mathrm{eV}$ ). The light curve shows maxima and minima with slow transitions between them.

Conclusions. To explain the gradual variations in the X-ray light curve and to avoid changes in absorption by neutral gas, a predominant part of the stellar wind must be ionised by the X-ray source. Compton scattering with variable electron column density (of the order of $5 \times 10^{24} \mathrm{~cm}^{-2}$ ) along the line of sight could then be responsible for the intensity changes. The X-ray variability of SMC 3 could also be caused by temperature changes in the hydrogen-burning envelope of the white dwarf, an effect that could even dominate if the stellar wind density is not sufficiently high.
\end{abstract}

Key words. stars: individual: SMC3 - binaries: symbiotic - X-rays: binaries - galaxies: individual: Small Magellanic Cloud white dwarfs

\section{Introduction}

The symbiotic star SMC 3 (Morgan 1992) in the Small Magellanic Cloud (SMC) was discovered as a super-soft source (SSS, Hasinger 1994) in X-rays during the ROSAT all sky survey (Kahabka \& Pietsch 1993). It is thought to be an interacting binary system, consisting of a cool M0 giant and a hot white dwarf (WD) in a wide orbit. In this model accretion from the stellar wind of the giant donor onto the WD leads to steady hydrogen burning on the WD surface that powers the high X-ray luminosity (Kahabka \& van den Heuvel 1997).

A series of ROSAT observations covering $\sim 6$ years (from Oct. 1990 to Nov. 1993 with the PSPC and from Apr. 1994 to Nov. 1996 with the HRI), revealed high X-ray variability (a factor of $\geq 80$ in ROSAT PSPC count rate), which was explained by an eclipse of the WD by the donor star (Kahabka 2004). This scenario needs obscuration of the X-ray emission region by the dense stellar wind close to the giant to account for the shape and the long duration of several months of the eclipse ingress and egress.
An optical outburst between December 1980 and November 1981 of up to $3 \mathrm{mag}$ in the $U$-band was reported by Morgan (1992). During this outburst no changes were detected in the I-band, and therefore, its origin was assigned to the hot stellar component. It is not clear whether the non-detection of SMC 3 with the Einstein satellite was due to X-ray inactivity before the optical outburst or to insufficient sensitivity (Kahabka 2004). The enrichment of nitrogen also suggests that there is evidence of a thermonuclear event (Vogel \& Morgan 1994). Results from modelling multi-wavelength data of SMC 3 with non-LTE models under the assumption of a constant X-ray source were presented in Orio et al. (2007) and Jordan et al. (1996). Orio et al. (2007) find that the variability cannot be caused by photoelectric absorption and suggested a "real" eclipse by the red giant.

SMC 3 was in a very luminous state during observation of field number 13 of the XMM-Newton (Jansen et al. 2001) large program SMC survey (Haberl \& Pietsch 2008). This enables spectral analysis of the EPIC-pn (Strüder et al. 2001) data with 
Table 1. XMM-Newton EPIC-pn observations of SMC 3.

\begin{tabular}{|c|c|c|c|c|c|c|c|c|c|c|c|}
\hline ObsID & $\begin{array}{c}\text { Satellite } \\
\text { revolution }\end{array}$ & Date & $\begin{array}{l}\text { Time } \\
\text { (UT) }\end{array}$ & Filter & $\begin{array}{l}\text { Net Exp } \\
{[\mathrm{s}]}\end{array}$ & $\begin{array}{l}\text { Net cts. } \\
(0.2-10.0\end{array}$ & $\begin{array}{l}\mathrm{Bg}^{a} \\
\mathrm{keV})\end{array}$ & $\begin{array}{l}\text { Net cts. } \\
(0.2-1.0\end{array}$ & $\begin{array}{r}\mathrm{Bg}^{a} \\
\mathrm{keV})\end{array}$ & $\begin{array}{r}R_{\text {sc }}^{b} \\
{\left[{ }^{\prime \prime}\right]}\end{array}$ & $\begin{array}{r}R_{\mathrm{bg}}^{b} \\
{\left[{ }^{\prime \prime}\right]}\end{array}$ \\
\hline 0301170501 & 1149 & 2006 Mar. 19 & $14: 45-20: 17$ & $\mathrm{mec}$ & $10446^{c}$ & 8287 & $12 \%$ & 8246 & $2 \%$ & 19 & 30 \\
\hline 0404680301 & 1344 & 2007 Apr. $11-12$ & $20: 00-02: 15$ & thin & 13986 & 170 & $13 \%$ & 137 & $5 \%$ & 16 & 50 \\
\hline 0503000201 & 1444 & 2007 Oct. 28 & $06: 11-11: 52$ & medium & 16607 & 451 & $9 \%$ & 407 & $4 \%$ & 21 & 40 \\
\hline 0601211301 & 1798 & 2009 Oct. 3 & $05: 31-14: 12$ & thin & 26535 & 39456 & $2 \%$ & 39338 & $1 \%$ & 69 & 55 \\
\hline
\end{tabular}

Notes. ${ }^{(a)}$ Ratio of background count rate to source count rate in the same energy band. ${ }^{(b)}$ Radius of the source and background extraction region. (c) No GTI screening was applied.

unprecedented statistical quality. Four spectra from different intensity states allow us now to study the spectral evolution of the hot component of SMC 3. We present the light curve starting with the first ROSAT detection in 1990, to investigate the nature of the variability seen from this system.

\section{Observations and data reduction}

SMC 3 was serendipitously observed four times with XMMNewton at off-axis angles between $8^{\prime}$ and $14^{\prime}$. Table 1 lists some details of the observations with the EPIC instruments operated in full-frame mode. In addition to the observation in Oct. 2009 from the SMC large survey program, we analysed three observations from 2006 and 2007 available in the archive. The first observation in March 2006 revealed SMC 3 in a high intensity state, but suffered from very high background. These data were used in the study of Orio et al. (2007). The two observations in April and October 2007 showed the source at low intensity. The detection of the source in the later observation was noted by Zezas \& Orio (2008).

To process the data, we used XMM-Newton SAS 10.0.0 ${ }^{1}$ with calibration files available until 17 June 2010, including the latest refinement of the EPIC-pn energy redistribution. As a standard, we selected good time intervals (GTIs) with an EPIC-pn

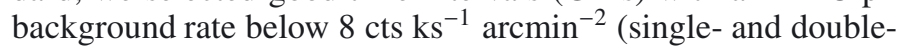
pixel events, 7-15 keV). However, for the observation in 2006 the background was between 500 and $2500 \mathrm{cts} \mathrm{ks}^{-1} \operatorname{arcmin}^{-2}$ and in order to retain any data, no background screening was applied. Since soft proton flares usually show a rather hard spectrum, the contribution to the super-soft spectrum of SMC 3 was still acceptable (cf. Table 1). The SAS task eregionanalyse was used to determine circular extraction regions by optimising the signal to noise ratio, as shown in Fig. 1 and listed in Table 1. We ensured that the source extraction region had a distance of $>10^{\prime \prime}$ to other detected sources. For the background extraction region, we chose a circle on a point source free area on the same CCD as the source. Since the MOS-spectra have lower statistical quality by a factor of 10 for such soft spectra and to avoid cross calibration effects between the EPIC instruments ${ }^{2}$, we are concentrating on the EPIC-pn spectra in this study. For the extraction of EPIC-pn spectra, we selected single-pixel events with FLAG $=0$. We binned the spectra to a minimum signal-to-noise ratio of 5 for each bin using the task specgroup.

\section{Spectral analysis of the EPIC-pn data}

We used xspec (Arnaud 1996) version 12.5.0x for spectral fitting. For all models, the Galactic photo-electric absorption was

\footnotetext{
${ }_{1}$ Science Analysis Software (SAS), http://xmm.esac.esa.int/ sas/

2 EPIC Calibration Status Document,

http://xmm2.esac.esa.int/external/xmm_sw_cal/calib/ index.shtml
}
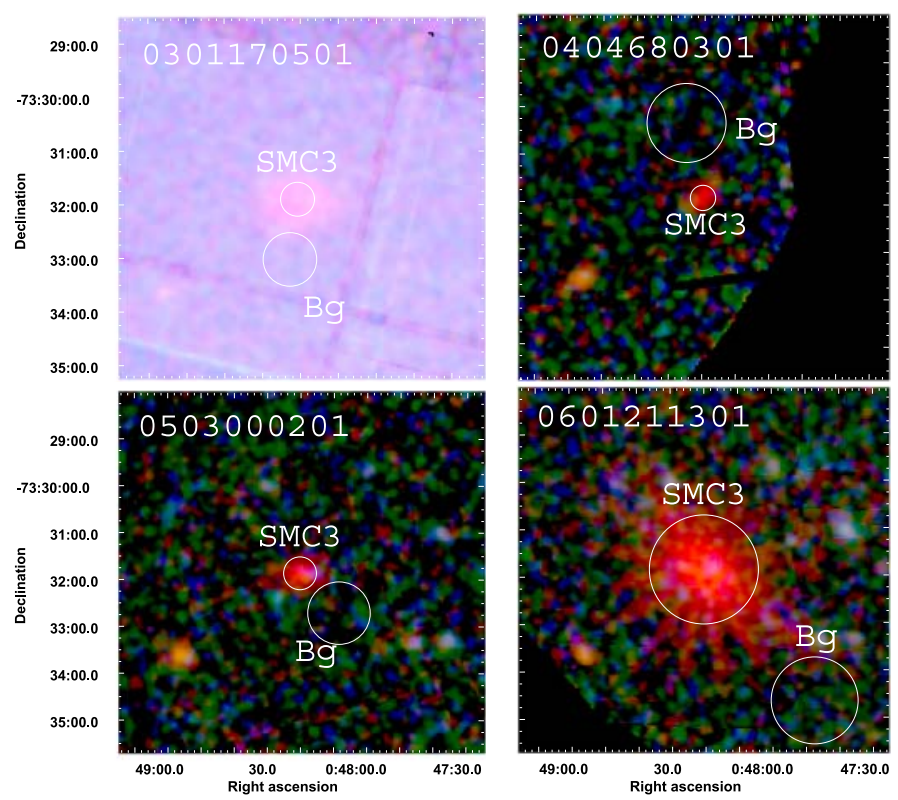

Fig. 1. Combined EPIC colour images of SMC 3 from the four XMMNewton observations. Red, green, and blue colours denote X-ray intensities in the $0.2-1.0,1.0-2.0$ and $2.0-4.5 \mathrm{keV}$ bands. Circles indicate the extraction regions. In the upper left, the background is increased at higher energies.

fixed at a column density of $N_{\mathrm{H}, \mathrm{gal}}=6 \times 10^{20} \mathrm{~cm}^{-2}$ (Dickey \& Lockman 1990) and elemental abundances according to Wilms et al. (2000), whereas the SMC column density was a free parameter with abundances at 0.2 for elements heavier than helium. At first, we investigated the recent EPIC-pn spectrum of 2009, which has unprecedented statistics compared to previous X-ray observations. For black-body emission, we obtain a best fit with $\chi^{2} /$ d.o.f. $=213 / 74$ with the best-fit parameters: $N_{\mathrm{H}, \mathrm{SMC}}=7.84_{-0.24}^{+0.44} \times 10^{20} \mathrm{~cm}^{-2}, k T=33.9 \pm 0.5 \mathrm{eV}$, and $\mathrm{a}$ bolometric luminosity of $L_{\text {bol }}=6.25_{-0.86}^{+1.08} \times 10^{38} \mathrm{erg} \mathrm{s}^{-1}$. This luminosity is super-Eddington (and leading to a WD radius $\sim 5$ 10 times larger than expected for a $1 M_{\odot} \mathrm{WD}$ ), which is often caused by the black-body approximation, as well as an overestimated temperature (see e.g. Greiner et al. 1991; Kahabka \& van den Heuvel 1997, and references therein). The residuals around $500 \mathrm{eV}$ (cf. Fig. 2) suggest contribution of nitrogen line emission, as observed in post-nova X-ray spectra of SSS (e.g. Rohrbach et al. 2009). The quality of the fit was improved to $\chi^{2} /$ d.o.f. $=119 / 72$ by including two Gaussian lines with fixed energy at $431 \mathrm{eV}$ (N vI) and $500 \mathrm{eV}$ (N viI) and line widths fixed to 0 . The equivalent widths of $19 \mathrm{eV}$ and $32 \mathrm{eV}$ for the $\mathrm{N}$ vi and $\mathrm{N}$ vII lines, respectively, are physically plausible, but the residuals could at least partially be also caused by calibration uncertainties. Alternatively, allowing the oxygen abundance in the SMC absorption component as free parameter also improved the 
Table 2. Results from the simultaneous black-body fit to the EPIC-pn spectra.

\begin{tabular}{lll}
\hline \hline $\begin{array}{c}\text { Model 1 } \\
T \text { and } L_{\mathrm{bol}} \text { constant with time } \\
N_{\mathrm{H} \text { variable }}\end{array}$ & \multicolumn{1}{c}{$\begin{array}{c}\text { Model 2 } \\
N_{\mathrm{H}} \text { and } T \text { constant with time } \\
L_{\mathrm{bol}} \text { variable }\end{array}$} & $\begin{array}{c}\text { Model 3 } \\
N_{\mathrm{H}} \text { constant with time } \\
\text { variable, } L_{\mathrm{bol}, i}=L_{\mathrm{bol}, 1}\left(T_{i} / T_{1}\right)^{4}\end{array}$ \\
\hline$k T=(32.5 \pm 0.5) \mathrm{eV}$ & $N_{\mathrm{H}}=(0.77 \pm 0.04) \times 10^{21} \mathrm{~cm}^{-2}$ & $N_{\mathrm{H}}=(0.77 \pm 0.02) \times 10^{21} \mathrm{~cm}^{-2}$ \\
$L_{\mathrm{bol}}=(8.8 \pm 1.6) \times 10^{38} \mathrm{erg} \mathrm{s}^{-1}$ & $k T=(33.7 \pm 0.5) \mathrm{eV}$ & $L_{\mathrm{bol}, 1}=(5.5 \pm 0.3) \times 10^{38} \mathrm{erg} \mathrm{s}^{-1}$ \\
$N_{\mathrm{H}, 1}=(1.08 \pm 0.05) \times 10^{21} \mathrm{~cm}^{-2}$ & $L_{\mathrm{bol}, 1}=(4.43 \pm 0.78) \times 10^{38} \mathrm{erg} \mathrm{s}^{-1}$ & $k T_{1}=(32.7 \pm 0.2) \mathrm{eV}$ \\
$N_{\mathrm{H}, 2}=(6.75 \pm 0.48) \times 10^{21} \mathrm{~cm}^{-2}$ & $L_{\mathrm{bol}, 2}=(0.12 \pm 0.02) \times 10^{38} \mathrm{erg} \mathrm{s}^{-1}$ & $k T_{2}=(24.3 \pm 0.3) \mathrm{eV}$ \\
$N_{\mathrm{H}, 3}=(6.91 \pm 0.23) \times 10^{21} \mathrm{~cm}^{-2}$ & $L_{\mathrm{bol}, 3}=(0.18 \pm 0.03) \times 10^{38} \mathrm{erg} \mathrm{s}^{-1}$ & $k T_{3}=(25.3 \pm 0.2) \mathrm{eV}$ \\
$N_{\mathrm{H}, 4}=(0.81 \pm 0.05) \times 10^{21} \mathrm{~cm}^{-2}$ & $L_{\mathrm{bol}, 4}=(6.47 \pm 1.15) \times 10^{38} \mathrm{erg} \mathrm{s}^{-1}$ & $k T_{4}=(33.8 \pm 0.4) \mathrm{eV}$ \\
$\chi^{2} /$ d.o.f $=940 / 154=6.10$ & $\chi^{2} /$ d.o.f. $=383 / 154=2.49$ & $\chi^{2} /$ d.o.f $=365 / 154=2.37$ \\
\hline
\end{tabular}

fit $\left(\chi^{2} /\right.$ d.o.f. $\left.=123 / 73\right)$, resulting in an oxygen abundance of 9.9 $9_{-2.7}^{+4.5}$ times solar with an $N_{\mathrm{H}, \mathrm{SMC}}=4.39_{-0.79}^{+0.66} \times 10^{20} \mathrm{~cm}^{-2}$. A hard spectral component, which could possibly be caused by the wind nebula, is not seen in the spectrum. Adding an apec plasma emission component to the black-body emission, with fixed temperature of $k T=500 \mathrm{eV}$ and SMC-abundances yields an upper limit for the emission measure of $E M=8.6 \times 10^{57} \mathrm{~cm}^{-3}$.

The derived values for the absorption $\mathrm{N}_{\mathrm{H}, \mathrm{SMC}}$ are well below the total SMC absorption in the direction of SMC 3 $\left(\sim 5 \times 10^{21} \mathrm{~cm}^{-2}\right.$, see Stanimirovic et al. 1999). This suggests that the symbiotic system is located on the near side of the large amount of Hi present in the SMC Bar.

We also tested non-local thermal equilibrium models provided by Thomas Rauch ${ }^{3}$ (Rauch \& Werner 2010). We found the best fit $\left(\chi^{2} /\right.$ d.o.f. $\left.=220 / 74\right)$ for a pure helium atmosphere with WD surface gravity $\log g=5, N_{\mathrm{H}, \mathrm{SMC}}=2.4 \times 10^{20} \mathrm{~cm}^{-2}$, $k T=6.9 \mathrm{eV}$, and $L_{0.2-1.0 \mathrm{keV}}^{\text {unabs. }}=1.57 \times 10^{37} \mathrm{erg} \mathrm{s}^{-1}$. For a pure hydrogen atmosphere with $\log g=9$ we found a best fit at $\chi^{2} /$ d.o.f. $=261 / 74$ with $N_{\mathrm{H}, \mathrm{SMC}}=1.1 \times 10^{21} \mathrm{~cm}^{-2}$, $k T=8.1 \mathrm{eV}$, and $L_{0.2-1.0 \mathrm{keV}}^{\text {unass. }}=2.59 \times 10^{38} \mathrm{erg} \mathrm{s}^{-1}$. If the spectrum in fact contains emission lines, this might be the reason of the inferior fit of the non-LTE models which produce spectra which are dominated by absorption lines from the white dwarf atmosphere. Since the black-body spectrum resulted in a better fit, we decided to use this model in our further investigations.

To study the spectral evolution of SMC 3, we fitted the EPICpn spectra of all four epochs simultaneously with a set of models based on absorbed black-body emission. In each model only one individual parameter for each spectrum and two common parameters for all spectra were allowed to vary (see below). Since the statistical quality of the high-flux spectrum is far better than for the other spectra, it also dominates the resulting $\chi^{2}$. This leads to relatively bad fits for the simple black-body models. Adding emission lines would improve the fits (see above). However, given the limited spectral resolution of the EPIC-pn instrument it is not clear if the lines have any physical meaning. Because we were mainly interested in the evolution of spectral parameters, we decided to use the simplest model.

Our first model (model 1 in Table 2) assumes temperature and luminosity not varying with time, while the absorbing column density can change with time. This corresponds to the eclipse model with varying absorption by the dense donor wind, as suggested by Kahabka (2004). This model gives an insufficient fit to the data (see Table 2). Although, the fit is statistically dominated by the two high-flux spectra, the spectral shape of the low-flux spectra cannot be reproduced by a high column density which predicts much less flux at lowest energies.

\footnotetext{
${ }^{3}$ http://astro.uni-tuebingen.de/ rauch/
}

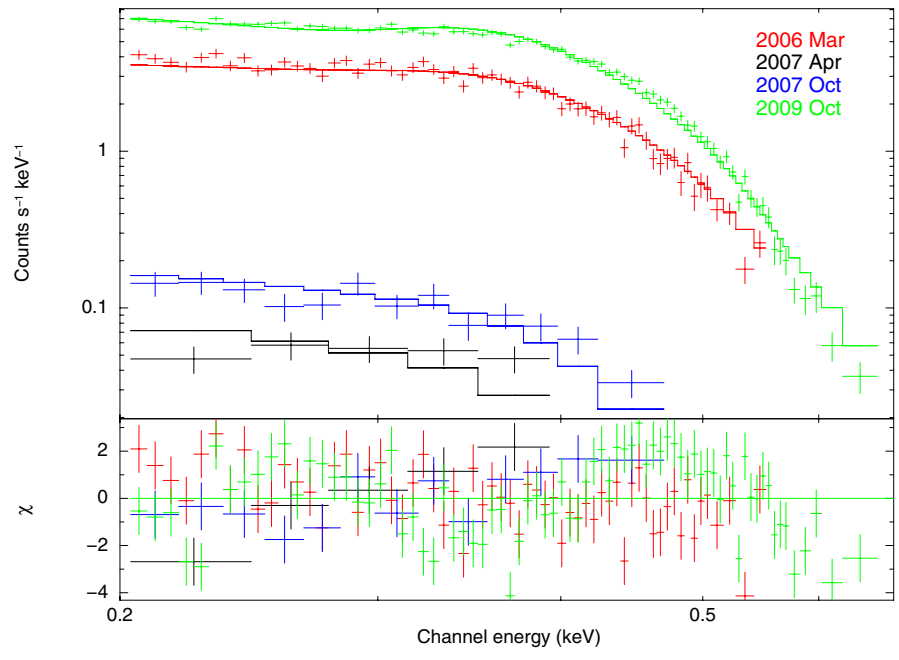

Fig. 2. EPIC-pn spectra of SMC 3 together with the best-fit black-body model 3 with variable temperature. No significant emission is seen above $0.7 \mathrm{keV}$.

For model 2, we fixed the spectral shape (same temperature and absorption) and allowed the luminosity to change (i.e. fitting individual normalisations which corresponds to a variable size of the emission area). This fits the data much better (Table 2). Within the systematic uncertainties the spectral parameters are in agreement with the black-body model of Orio et al. (2007).

An even better fit was achieved by our third model with varying source temperature. The corresponding black-body luminosities were related to the temperature $\left(L_{\mathrm{bol}} \propto T^{4}\right)$. The results for this model are again described in Table 2 and the individual spectra with the model fit are plotted in Fig. 2.

\section{The X-ray light curve of SMC 3}

To analyse the temporal behaviour of the system, we reconciled the X-ray light curve of SMC 3 starting from the first detection by ROSAT in 1990 . To convert the ROSAT count rates, provided by Kahabka (2004), into fluxes, we simulated a ROSAT PSPC spectrum based on the spectral model derived from the simultaneous fit with variable normalisation and the PSPC detector response. We obtained a conversion factor of $1.54 \times 10^{-11} \mathrm{erg} \mathrm{cm}^{-2} \mathrm{cts}^{-1}$. All fluxes are computed for the $0.2-1.0 \mathrm{keV}$ band. Because of the dominant statistics of the XMM-Newton high-flux spectrum in the model fit, this factor rather corresponds to the high-flux state. Using the model with variable temperature, the conversion factor for the low flux can be lower by a factor of $\lesssim 2$. Analogous, for 


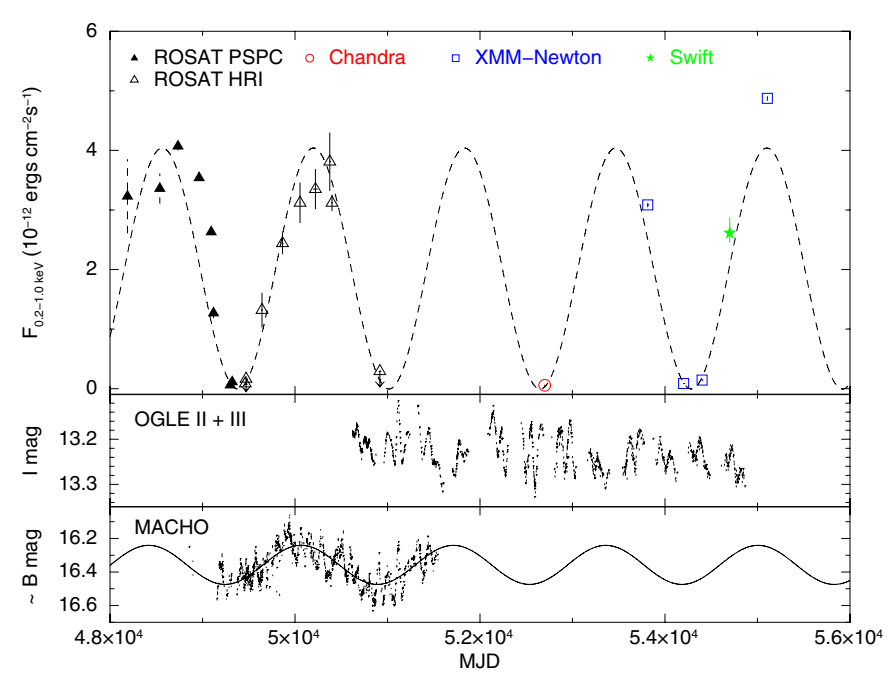

Fig. 3. The $0.2-1.0 \mathrm{keV} X$-ray light curve of SMC 3 (upper panel), the light curves in the $I$-band (OGLE, middle panel) and the approximate $B$-band (MACHO, lower panel). The dashed line shows the best-fit sine function to the X-ray light curve and the solid line the best-fit sine function to the MACHO light curve (see text).

the ROSAT HRI, this simulation yields a conversion factor of $4.63 \times 10^{-11} \mathrm{erg} \mathrm{cm}^{-2} \mathrm{cts}^{-1}$.

We searched the Swift archive for observations covering SMC 3. The source was detected in observation 00037787001 with $\sim 3$ ks exposure on 18 August 2008. The source and background spectrum was extracted with xselect and the effective area file was created using xrtmkarf. The resulting spectrum contains 135 net counts, which is insufficient to distinguish between the above described models. Thus we fitted only the normalisation and assumed the spectral shape according to the simultaneous fit to the EPIC-pn spectra with variable normalisation. This fit yields a flux of $2.61_{-0.15}^{+0.26} \times 10^{-12} \mathrm{erg} \mathrm{cm}^{-2} \mathrm{~s}^{-1}$.

To derive XMM-Newton fluxes, we integrated the best-fit model with variable temperature as described above. The source flux during a Chandra observation in February 2003 was deduced from the parameters of the best-fit black-body model reported by Orio et al. (2007).

Figure 3 shows the X-ray light curve of SMC 3 since the first detection by ROSAT in 1990. By modelling the light curve with several eclipses, we realised, that (i) the transition from high to low intensity occurs over a long time period and that (ii) the time scales of the duration of the high and low intensity intervals are of the same order. Thus, instead of eclipses the light curve may also be interpreted by several periodic outbursts. For demonstration, the dashed line in Fig. 3 shows a fit with a sine function. To account for uncertainties in the flux conversion and cross calibration between the different instruments we included a $20 \%$ systematic error on the flux. As best-fit ephemeris for the X-ray minimum we then obtain ( $90 \%$ confidence errors):

$\mathrm{MJD}_{\text {min }, \mathrm{x}}=(49382 \pm 10)+N \times(1634 \pm 7)$ days.

The relatively high flux measured in the last XMM-Newton observation might suggest possible changes in the amplitude of the modulation.

\section{MACHO and OGLE data}

The OGLE-II (Udalski et al. 1997) and OGLE-III (Udalski et al. 2008) $I$-band as well as the MACHO $B$-band light curves are shown in the lower two panels of Fig. 3. The calibrated MACHO light curve was shifted in magnitude to match its average $B$ magnitude with that measured by Zaritsky et al. (2002). In the $I$-band of OGLE-II and the $B$-band of MACHO, Kahabka (2004) found correlating quasi-periodic oscillations with periods around 110 days which might be related to pulsations of the red giant star. These short variations are also present in the OGLE-III data. With the OGLE-III data, which cover a much longer time interval, we can now rule out a significant variation with the 1630 day cycle as suggested by the X-ray light curve. As noted by Kahabka (2004), the MACHO light curve shows a quasi sinusoidal modulation with a period of $\sim 4$ years, in addition to shorter variations. A fit of a sine function to the MACHO $B$-band data (solid line in Fig. 3), results in an ephemeris for the optical minimum of

$$
\mathrm{MJD}_{\min , \mathrm{B}}=(49242 \pm 9)+N \times(1647 \pm 24) \text { days }
$$

To account for the short-term variations we added a systematic error of 0.05 mag to the $B$-band magnitudes. Formally, the fits to $\mathrm{X}$-ray and MACHO light curves indicate a phase shift of (140 \pm 14) days (optical preceding the $X$-rays) while the periods agree within the errors. However, it should be noted that the MACHO light curve covers only $\sim 1.5$ cycles and is superimposed by the short term variations which may influence the results.

\section{Discussion}

SMC 3 was observed with XMM-Newton at four epochs, covering the super-soft X-ray source twice at high and twice at low intensity. A strong variation in the X-ray flux by a factor of more than $\sim 50$ between minimum and maximum intensity is found in the $0.2-1.0 \mathrm{keV}$ band. We showed, that the light curve can qualitatively be described by a sine function with a period of 1633 days. This simple model can only be a crude approximation of the light curve, but the $\sim 20$ year coverage indicates a high regularity of the period with similar duration of high and low intensity intervals. The regularity of the X-ray light curve, with meanwhile four observed minima, strongly supports the interpretation of the 4.5 year period as the orbital period of the binary system. Assuming masses of $2 M_{\odot}$ and $1 M_{\odot}$ for the Mgiant and the white dwarf, respectively, the orbital period implies a semi-major axis of the binary system of 3.9 AU.

We analysed the spectral evolution and found, that the variability of the X-ray flux cannot be explained by photo-electric absorption by neutral gas with varying column density. To avoid the strongly energy-dependent attenuation of soft X-rays, Kahabka (2004) discussed absorption due to highly ionised gas. In this picture the strong X-ray source ionises the stellar wind around it. Compton scattering on free electrons would then reduce the X-ray flux along the line of sight most efficient when looking through the dense innermost regions near the M-star. This mimics variable intensity with little energy dependence (no significant change of spectral shape). Using a Compton scattering model (cabs in xspec), instead of variable normalisation, would require a column density of $>4.8 \times 10^{24} \mathrm{~cm}^{-2}$ (completely ionised absorber) to reduce the X-ray intensity from maximum to minimum. In this picture scattering of $\mathrm{X}$-rays into the line of sight is neglected or at least assumed not to change significantly between the two states. Using the estimated mass, size and density of the ionised wind region as given by Orio et al. (2007) yields a column density to its centre of $5.8 \times 10^{23} \mathrm{~cm}^{-2}$. This is a factor of $\sim 8$ lower than our estimate from Compton scattering and may be explained by the simplified assumption of a constant wind density while the line of sight during the low intensity observations should pass through the denser wind regions 
near the giant star. In this picture most of the stellar wind must be ionised, consistent with the fact that we do not see a variable contribution of photo-electric absorption by neutral gas. In this model, the low intensity can still be explained by an eclipse of the X-ray source by the giant star and its stellar wind. An eclipse by the star only would be short ( $\sim 10 \%$ of the orbital period) with sharp ingress and egress while the dense inner wind regions cause a long gradual eclipse ingress (and egress) by increasing (decreasing) Compton scattering along the line of sight. This geometric model with scattering in the circum-stellar material is consistent with both the gradual ingress/egress of the $\mathrm{X}$-ray light curve and the spectral changes (no significant variation in photo-electric absorption). The exact shape of the light curve should then depend on the geometry of the binary system and the distribution of free electrons in the stellar wind.

Our spectral analysis shows, that the X-ray variability can alternatively be dominated by temperature changes, varying between 24 and $34 \mathrm{eV}$. Assuming a constant size of the emitting area, this corresponds to a variation in $L_{\text {bol }}$ (for spherically symmetric emission) by a factor of 4.3. The larger variation in observed instrumental count rates would then be caused by shifting the spectra with lower temperature out of the sensitive energy band of EPIC. This behaviour is independent of the model used. The spectra show clear softening which is parametrised by a temperature change (similar if black-body or atmosphere models are used). A possible scenario might be an elliptical orbit of the white dwarf around the M-giant (or equatorial mass ejection with inclined WD orbit), causing accretion at different rates. Variable accretion, even at low level, can lead to large temperature changes in the burning layer (Paczynski \& Rudak 1980). Similar scenarios were used to explain X-ray variability in other SSS (e.g. AG Dra, Greiner et al. 1996). However, we note, that in those cases usually an anti-correlation of X-ray and optical luminosity is observed, whereas in the case of SMC 3 these two are clearly correlated.

Assuming the same temperature and absorption (and no change in Compton scattering) for the low and high intensity spectra, the inferred radii would be different by a factor of $\sim 8$ to account for the factor of 60 difference in $L_{\mathrm{bol}}$. In general for stable shell burning on the WD surface, an increase of the hydrogen burning envelope (e.g. due to a higher accretion rate) leads to an increase of both temperature and radius (Fujimoto 1982). Increasing temperature and declining Compton scattering both lead to an increasing X-ray luminosity. It depends on the orientation of the orbit with respect to the observer, how much the two effects act in phase. Additional temperature variations may therefore reduce or increase the amount of Compton scattering required to explain the X-ray luminosity variations.

Superimposed on the general long term variation in the X-ray light curve, we probably see effects imposed by the donor star. The $\sim 110$ days brightness variations seen in the $I$-band suggests changes in the stellar wind which can lead to variations in the mass accretion rate onto the white dwarf. Since the 1630 day $\mathrm{X}$-ray period is not visible in the $I$-band, it is unlikely that this period is caused by the cool stellar component, which seems to remain rather unaffected by the process producing this variation. In the $B$-band, both modulations are seen, the 1630 day period derived from the X-rays and the $\sim 110$ day variations which correlate with the $I$ mag (as already pointed out by Kahabka 2004). Therefore, the cool companion star and the region where the $\mathrm{X}$-ray emission is produced most likely both contribute to the $B$-band. If viewing effects produce the variation in $B$ in a similar way as in the X-rays (by changing extinction) or if heating of the cool star by the X-ray source is causing this variation remains unclear: While Kahabka (2004) finds X-ray heating insufficient to account for the observed $B$-band modulation, Orio et al. (2007) discuss irradiation effects influencing the mass outflow as very important.

\section{Conclusions}

The new X-ray data of SMC 3 show that two scenarios can qualitatively explain the spectral evolution and the shape of the light curve. The evolution of the X-ray spectra is incompatible with changing photo-electric absorption by neutral gas, but is consistent with energy-independent intensity and/or with temperature variations. As suggested before by Kahabka (2004), Compton scattering in a predominantly ionised stellar wind could lead to the observed intensity variations if the stellar wind density (mass loss rate) is high enough. Additionally, temperature changes in the burning layer of the WD, which are caused by variable accretion, can reduce the required wind densities.

Acknowledgements. This publication is partly based on observations with $X M M-N e w t o n$, an ESA Science Mission with instruments and contributions directly funded by ESA Member states and the USA (NASA). The XMMNewton project is supported by the Bundesministerium für Wirtschaft und Technologie/Deutsches Zentrum für Luft- und Raumfahrt (BMWI/DLR, FKZ 50 OX 0001) and the Max-Planck Society. R.S. acknowledges support from the BMWI/DLR grant FKZ 50 OR 0907. This paper utilises public domain data obtained by the MACHO Project, jointly funded by the US Department of Energy through the University of California, Lawrence Livermore National Laboratory under contract No. W-7405-Eng-48, by the National Science Foundation through the Center for Particle Astrophysics of the University of California under cooperative agreement AST-8809616, and by the Mount Stromlo and Siding Spring Observatory, part of the Australian National University. The OGLE project has received funding from the European Research Council under the European Community's Seventh Framework Programme (FP7/2007-2013)/ERC grant agreement No. 246678 to AU.

\section{References}

Arnaud, K. A. 1996, in Astronomical Data Analysis Software and Systems V, ed. G. H. Jacoby, \& J. Barnes, ASP Conf. Ser., 101, 17

Dickey, J. M., \& Lockman, F. J. 1990, ARA\&A, 28, 215

Fujimoto, M. Y. 1982, ApJ, 257, 752

Greiner, J., Hasinger, G., \& Kahabka, P. 1991, A\&A, 246, L17

Greiner, J., Bickert, K., Luthardt, R., et al. 1996, in Supersoft X-Ray Sources, ed. J. Greiner, Lecture Notes in Physics (Berlin: Springer Verlag), 472, 267

Haberl, F., \& Pietsch, W. 2008, in X-rays From Nearby Galaxies, ed. S. Carpano, M. Ehle, \& W. Pietsch, 32

Hasinger, G. 1994, in Rev. Mod. Astron., ed. G. Klare, 7, 129

Jansen, F., Lumb, D., Altieri, B., et al. 2001, A\&A, 365, L1

Jordan, S., Schmutz, W., Wolff, B., Werner, K., \& Muerset, U. 1996, A\&A, 312, 897

Kahabka, P. 2004, A\&A, 416, 57

Kahabka, P., \& Pietsch, W. 1993, in New Aspects of Magellanic Cloud Research, ed. B. Baschek, G. Klare, \& J. Lequeux, Lect. Not. Phys. (Berlin: Springer Verlag), 416, 71

Kahabka, P., \& van den Heuvel, E. P. J. 1997, ARA\&A, 35, 69

Morgan, D. H. 1992, MNRAS, 258, 639

Orio, M., Zezas, A., Munari, U., Siviero, A., \& Tepedelenlioglu, E. 2007, ApJ, 661,1105

Paczynski, B., \& Rudak, B. 1980, A\&A, 82, 349

Rauch, T., \& Werner, K. 2010, Astron. Nachr., 331, 146

Rohrbach, J. G., Ness, J., \& Starrfield, S. 2009, AJ, 137, 4627

Stanimirovic, S., Staveley-Smith, L., Dickey, J. M., Sault, R. J., \& Snowden, S. L. 1999, MNRAS, 302, 417

Strüder, L., Briel, U., Dennerl, K., et al. 2001, A\&A, 365, L18

Udalski, A., Kubiak, M., \& Szymanski, M. 1997, Acta Astron., 47, 319

Udalski, A., Szymanski, M. K., Soszynski, I., \& Poleski, R. 2008, Acta Astron., 58,69

Vogel, M., \& Morgan, D. H. 1994, A\&A, 288, 842

Wilms, J., Allen, A., \& McCray, R. 2000, ApJ, 542, 914

Zaritsky, D., Harris, J., Thompson, I. B., Grebel, E. K., \& Massey, P. 2002, AJ, 123,855

Zezas, A., \& Orio, M. 2008, The Astronomer's Telegram, 1379, 1 